Revue d'histoire de l'Amérique française

Q4 REVUE D'HISTOIRE DE L'AMÉRIQUE FRANÇAISE

\title{
Colonisation au Canada sous Talon
}

\section{Lionel Groulx}

Volume 4, numéro 1, juin 1950

URI : https://id.erudit.org/iderudit/801616ar

DOI : https://doi.org/10.7202/801616ar

Aller au sommaire du numéro

\section{Éditeur(s)}

Institut d'histoire de l'Amérique française

\section{ISSN}

0035-2357 (imprimé)

1492-1383 (numérique)

Découvrir la revue

\section{Citer cet article}

Groulx, L. (1950). Colonisation au Canada sous Talon. Revue d'histoire de l'Amérique française, 4(1), 61-73. https://doi.org/10.7202/801616ar d'utilisation que vous pouvez consulter en ligne.

https://apropos.erudit.org/fr/usagers/politique-dutilisation/ 


\section{COLONISATION AU CANADA SOUS TALON}

Vers la fin de l'intendance de Jean Talon, à quel point s'en trouve la colonisation au Canada? L'intendant y a mis tout son entrain, tout son dynamisme. Il ne cesse de réclamer des récompenses, des marques de distinction pour les hommes d'initiative, les actifs qui font avancer la colonie. Talon aura eu ce mérite, assez rare parmi les gouvernants, de tenter le possible pour intéresser les petits gentilshommes de France, les grands riches, mais "sans qualite", à la colonisation du pays. Pour présenter l'amorce toute-puissante à cette sorte de gens, il eât voulu amener le roi a "tiltrer icy des terres de Chastelenie, Baronnie, Vicomté, et autres de plus grand caractère..." ${ }^{1}$ Le roi cède presque toujours aux sollicitations de l'intendant; il consent a dépenser de fortes sommes; par distribution de médailles et même de parchemins nobiliaires aux méritants, il fait ce qu'il peut pour stimuler, encourager les faiseurs de terre. La persuasion est bien établie dans la colonie que le meilleur moyen de gagner la faveur royale, c'est de s'appliquer a défricher. Dans les lettres de noblesse de Nicholas Juchereau de SaintDenis, le premier Juchereau est cité en exemple, parce que "au lieu de faire commerce comme beaucoup d'autres personnes, yl fut des premiers qui s'attacherent uniquement... à faire des establissements assez considerables et a faire travailler au deffrichement et a la culture des terres..." ${ }^{2}$ D'autres, pour les mêmes motifs, s'attirent les mêmes grâces. ${ }^{3}$

Les résultats ont-ils répondu à ces attentions de l'intendant et du roi? Tous deux viennent de tenter une expérience quelque peu

1. Rapport de l'Archiviste de la Province de Québec pour 1990-1991 (RAPQ), 86, 96, 129, 148, 154.

2. Pierre-Georges Roy, Archives de la Province de Quebec: Lettres de noblesse, généalogies, érections de comtés et baronnies insinuées par le Conseil souverain de la Nouvelle-France (2 vol., Beauceville, 1920), I: 38.

3. P.-G. Roy, Ibid., I: 115, 123-124. - Aussi P.-G. Roy, La famille Godefroy de Tonnancour (Lévis, 1904), 9. 
téméraire. Ils n'avaient pas à s'inquiéter du travail des anciens colons et des immigrants venus des milieux agricoles de France. Majs les autres, les militaires du régiment de Carignan qu'on venait de licencier? Qu'attendre de ces officiers de carrière, invités, non pas à l'exploitation d'un domaine terrien, mais à l'établissement de ce domaine, c'est-à-dire à une création de toutes pièces, et à l'aide de défricheurs qui sont eux-mêmes des soldats? Malheureusement, pour établir ce point d'histoire, les pièces d'archives mises au jour n'abondent guère. Entre les années 1668 et 1679 les recensements officiels font défaut. Les premiers aveux et dénombrements sont rares et plutôt laconiques. De "papier-terrier" pour la période, nous ne possédons que celui de la Compagnie des Indes occidentales qui couvre les seules années 16671668. " L'autre, le "Papier-Terrier", ordonné par le roi et travail à n'en plus finir, ne viendra que beaucoup plus tard. Ce n'est pas avant 1740 , comme l'on sait, que le procureur général Verrier mettra le dernier point au $7^{\mathrm{e}}$ volume, et encore y manquera-t-il un supplément. ${ }^{5}$ Il reste, en utilisant quelques autres sources documentaires, à tenter un bilan quelque peu approximatif.

Un état envoyé en France en 1668 permet de relever, pour cette année-là, 15,642 arpents en culture, soit une augmentation d'environ 4,000 arpents sur l'année précédente. Mise au courant de ces progrès la Mère de l'Incarnation s'écrie qu' 'il est incroyable combien ce pays se découvre et se peuple partout." 6 Talon ne parle pas autrement en 1667 lorsqu'il affirme que le "pays a bien changé de face quant aux habitations et à la découverte des terres," qu' "il commence à prendre une autre forme que celle qu'il avoit avant l'arrivée des troupes du Roy", et qu'il "se découvre en beaucoup d'endroicts..." ' Le sol garde

4. Papier-terrier de la Compagnie des Indes occidentales, 1667-1668, publié par Pierre-Georges Roy (Beauceville, 1931).

5. Hocquart au ministre, 4 octobre 1740, AC, C 11A, 73: 83.

6. Lettres de la Révérende Mère Marie de l'Incarnation (née Marie Guyard) première supérieure du Monastère des Ursulines de Québec, Nouvelle édition... par l'abbé Richaudeau (2 vol., Paris, 1876), II: 314, lettre à son fils, 29 octobre 1665.

7. RAPQ (1930-1931), 81-90. 
sa réputation de fertilité. Les seigneurs de l'entourage de $M$. de Tracy, qui arrivent des Antilles, trouvent le Canada d'"un séjour ravissant en comparaison des îles d'Amérique". Ils admirent la générosité de la terre, terre où "les blés, les légumes et toutes sortes de grains croissent en abondance... terre à froment, laquelle plus on la découvre des bois, plus elle est fertile et abondante". ${ }^{8}$ Encore en 1668 , la récolte des grains s'élève à 130,978 minots. ${ }^{9}$ En 1673 un seigneur, M. Berthelot, se dit en état de fournir 5,000 quintaux de pois pour l'approvisionnement de la marine française. ${ }^{10}$ En 1664 la colonie récoltait déjà un surplus de blé. ${ }^{11}$ En 1670 Talon renvoie aux Antilles cent barriques de farine de France pour permettre, à meilleur prix, dans la colonie, l'écoulement de la surabondance de blé. ${ }^{2}$ Car il y a déjà des années que les colons ne savent que faire du surplus de leur récolte. ${ }^{13}$ Le lin, le chanvre surtout, réussissent assez bien, pour fournir du travail aux métiers. Pour stimuler la culture du chanvre, Talon achète une part de la production. ${ }^{14}$ Une culture, celle du tabac, s'est vu refuser l'encouragement royal, par crainte d'une concurrence inopportune au tabac des Antilles; d'autres entreprises paraissent d'ailleurs plus utiles à $\mathbf{S a}$ Majesté. ${ }^{15}$ Les potagers se développent; des fermes, parmi les plus anciennes, accroissent leurs vergers. Les Sœurs Hospitalières de Québec possèdent "un verger de plus beaux qu'on pourrait voir en France." 16 Malheureusement, le terrible hiver de 1670 qui maintient de la glace dans les jardins jusqu'au mois de juin, fait parmi les arbres fruitiers de terribles ravages. ${ }^{17}$ Le cheptel marque, lui aussi, des progrès notables.

8. Lettres de la Révérende Mère Marie de l'Incarnation, éd. Richaudeau, II: 313.

9. Censuses of Canada. 1665 to 1871. Recensements du Canada. Statistiques du Canada (Ottawa, 1876), IV: 8.

10. RAPQ (1930-1931), 177.

11 Jugements et délibérations du Conseil Souverain de la Nouvelle-France (6 vol., Québec, 1885-1891), I: 205.

12. En 1670 l'intendant avait apporté de France 150 milliers de farine et 200 barils de lard. AC, B 2 (1670): 38 .

13. RAPQ (1930-1931), 136. Relations des Jésuites (RJ) éd. Thwaites (73 vol., Cleveland, 1896-1901), LI: 174 .

14. RAPQ (1930-1931), 69, 112, 160.

15. RAPQ (1930-1931), 160, 169.

16. Lettres de la Révérende Mère Marie de l'Incarnation, êd. Richaudeau, II: 449.

17. Idem, II: 50 . 
De 1665 a 1668 le roi envoie 91 chevaux et 80 moutons. ${ }^{18}$ Les chevaux se reproduisent si rapidement qu'en 1670 , l'on en peut faire commerce. ${ }^{19}$ L'état de 1668 recense 3,400 bêtes à cornes. Vers 1670 la colonie est en état de se passer des lards de la Rochelle, d'où l'on en tirait jusqu'à 800 barils par année. Cette année-là même, Talon fait charger pour les Antilles 400 barils de lard venus de France, afin de laisser le marché libre aux lards du pays. ${ }^{2}$ Talon annonce triomphant que l'Acadie et la Nouvelle-France pourront bientôt fournir aux Antilles leur provision de viande salée. Bref, par ses céréales, blés, légumes, par son cheptel, par ses pêches, le Canada a gagné une telle avance qu'il suffit à ses besoins et peut même exporter de sa surproduction. ${ }^{21}$ Des pauvres se rencontrent, sans doute, dans la colonie. Il faut à une famille deux ou trois années, dira la Mère de l'Incarnation, "avant que d'avoir de quoi se nourrir, sans parler du vêtement, des meubles et d'une infinité de petites choses nécessaires à l'entretien d'une maison." 22 En attendant, le colon peut suppléer à ses grains, à ses légumes, par les produits de la chasse, toujours abondante en hiver. Pour se procurer des vêtements, des ustensiles, il débite du bois de charpente toujours en demande et au prix fort. Ainsi, en peu d'années, pour peu que l'on ait de la conduite, continue l'Ursuline, on connaît une modeste aisance, on devient même riche, "autant qu'on le peut être dans un pays nouveau comme est celui-ci". ${ }^{23}$ La modeste aisance, elle apparait dans le perfectionnement relatif de l'outillage des fermes. Les défricheurs n'en sont plus à ne remuer le sol qu'avec la pioche et la bêche. Un inventaire des biens de Mlle Mance établit, par exemple, que sur l'une des terres de l'Hôtel-Dieu de Ville-Marie, on fait usage de deux charrues à roues, "charues garnies, avec leurs essieux de fer, les roues non ferrées". L'inventaire recense aussi une herse "a cheville de bois"; la ferme possède cinq bœufs de labour: bœufs de labour que le cheval est en train de remplacer. ${ }^{2} 4^{3}$ On se rappelle que de 1665 a 1668 , le

18. RAPQ (1930-1931), 106.

19. RAPQ (1930-1931), 128.

20. RAPQ (1930-1931), 136, 161.

21. RAPQ $(1930-1931), 120,135,161,164$.

22. Lettres de la Révérende Mère Marie de l'Incarnation, éd. Richaudeau, II: 313-314.

23. Ibid., II: 313-314.

248. M.-Claire Daveluy, Jeanne Mance (Montréal, 1934), 302-303. 
roi a pu faire passer au Canada 91 chevaux. "On nous a donné pour notre part deux belles cavales et un cheval, tant pour la charrue que pour le charroi", écrit en France la Mère de l'Incarnation. ${ }^{24}$

L'aisance se manifeste encore au prix que prennent déjà quelques propriétés terriennes. En 1668, les 160 arpents de terre de feu Pierre le Gardeur de Repentigny sont évalués à huit mille livres. ${ }^{20}$ Vers ce même temps, les Sœurs de l'Hôtel-Dieu de Québec vendent deux seigneuries héritées de Madame d'Aillebout, les Grondines et Argentenay, la première, 1,000 écus, la seconde pour 100 livres de rente. ${ }^{25}$ En 1670 Michel Gamelain vend 2,700 livres la seigneurie de Sainte-Anne de la Pérade $^{26}$. En 1666 le Père Beschefer, déjà étonné de l'aspect du Canada, se plaît à le dire "pas si sauvage que l'on s'est imagine". "Dans les habitations françaises", assure le jésuite, l'on trouve "presque les mesmes douceurs qu'en Europe et les tables des personnes qui ont de l'argent à y dispenser sont aussy bonnes qu'en France." 27

On voudra savoir sur quels points de la colonie, se manifeste plus victorieusement ce progrès. Comme il va de soi, c'est aux environs des anciens postes qu'apparaît plus visiblement l'avance des défricheurs. "Il n'y a proprement de lieux ramassés", constate l'abbé Fénelon, "que Québec, le Cap, les Trois-Rivières, la ville de Montréal et quelques villages que $M$. Talon a fait faire à la vue de Québec." 28 La région de Québec, siège de la capitale et terme maintenant de la navigation transatlantique, absorbe naturellement le plus gros des immigrants. Pour la ville elle-même, les chroniqueurs n'échappent pas à

24b. Lettres (éd. Richaudeau) II: 352.

24c. Les Ursulines de Québec depuis leur établissement jusqu'd nos jours (4 vol., Québec, 1863-1866), I: 284, note.

25. Les Annales de l'Hôtel-Dieu de Québec 1613-1716, éditées par Dom Albert Jamet, (Québec, 1939), 176.

26. Raymond Douville, Les premiers Seigneurs et colons de Sainte-Anne de la Perade (1667-1681) (Les Trois-Rivières, 1946), 39.

27. RJ, L: 171-172. Voir encore: "Notes sur la famille Bazire", Bulletin des recherches historiques (BRH) (février 1936), 65-70.

28. Mémoire inédit de l'abbé Fénelon, 1671, AC, C IIA, 3: 236-303. Voir l'abbé H.-A. Verreau, Les deux Abbés de Fénelon (Lévis, 1898). 
ce que j'oserais appeler une sorte d'emballement. La capitale ressemble tout au plus aux gros bourgs et aux petites villes de province, dans la France de l'époque. On n'y est guère particulier sur l'hygiène et la propreté. La rue sert trop souvent de dépotoir. Encore en 1664, il a fallu une ordonnance du Conseil souverain pour empêcher les habitants de la basse-ville de jeter paille et fumier sur le devant de leur porte. ${ }^{29} \mathrm{~A}$ son arrivée, Frontenac ne peut se défendre d'admiration devant le site naturel de Québec: "Rien ne m'a paru si beau et si magnifique que la situation de la ville de Québec qui ne pourroit pas être mieux postée quand elle devrait devenir un jour la capitale d'un grand empire." Mais l'arrivant n'en est pas moins suffoqué par l'absence de tout souci d'urbanisme dans le développement de la ville; les maisons y ont poussé dans le plus parfait désordre, sans la moindre symétrie. ${ }^{30}$ Voyez pourtant comme on prend quand même et facilement le ton lyrique: "Jugez de là que Québec est quelque chose de considérable", s'exclame, demi-plaisant, sans doute, le Père Beschefer. Et il nous décrit la capitale: "La Ville basse est bâtie sur le bord de l'eau et de marée haute... Il y a quelques maisons assez considerables. On y en bastit deux l'an passée dont l'une a esté vendue 22 milles livres et l'autre en vaut bien 15 mille. Habitent là les marchands et le gros de la population." Et voici la haute ville, séjour des fonctionnaires, du clergé et des communautés. Elle n'est considérable que par les Églises et les maisons religieuses: église cathédrale, "tres bien ornée". "Nous avons cette année commencé une église... qui a 100 pieds de long et 30 de large... Les religieuses ursulines et hospitalieres sont bien basties. En un mot les églises sont icy comme dans les bonnes villes de France." 31

Mais, au vrai, quelle métamorphose dans le paysage. A proximité de la ville, au delà de la rivière Saint-Charles, Talon met sa bouillante activité à pousser le défrichement de sa future baronnie des Islets. Dans le seul été de 1668, Bourg-Talon surgit dans la forêt. BourgRoyal, Bourg-la-Reine suivent bientôt, destinés à devenir les joyaux de la future baronnie. ${ }^{32}$ De sa maison située au haut de la côte de la

29. Jugements et délibérations du Conseil souverain, op. cit., I: 186-187.

30. RAPQ (1926-1927), 11-2.

31. RJ, L: 172-174.

32. RAPQ (1930-1931), 56, 129. C'est par lettres patentes du 14 mars 1671 que le roi incorporait les trois bourgs à la seigneurie des Islets et érigeait le tout en baronnie. (Jugements et délibérations du Conseil souverain, I: 692). 
montagne, ${ }^{3} 3$ Talon et chacun avec lui peut apercevoir, sans doute, les vastes granges où l'intendant élève chevaux, bovins et autre bétail, sa large basse-cour remplie de coqs-d'inde, de canards, d'outardes, d'oies, de pigeons, etc., dont il fait l'élevage pour fournir aux colons de bons sujets de reproduction. On aperçoit aussi, sans doute, sa houblonnière, six mille perches qui font, dans la seigneurie, une enclave de verdure. ${ }^{34}$ En fait la baronnie est devenue, parmi les seigneuries, "une des plus considérables du pays." ${ }^{35}$ Sur la carte de Gédéon de Catalogne, dressée en 1709, on distingue tout de suite, pour leur forme géométrique originale, ces trois créations de Talon: deux carrés et un rectangle, avec chacun un foyer central d'où les terres des colons s'irradient en forme étoilée. A Notre-Dame des Anges, seigneurie des Jésuites, le développement n'est pas moins sensible. Les aveux et dénombrements de la seigneurie qu'on peut consulter aux Archives provinciales de Québec, font foi des nombreuses concessions de terre accordées aux colons. ${ }^{36}$ L'Ile d'Orléans, élevée en 1676 à la dignité de Comté de Saint-Laurent, en faveur de François Berthelot, ${ }^{37}$ compte, vers ce temps-là, plus de mille habitants repartis en quatre paroisses et plus de 2,000 arpents de terre en culture. ${ }^{38} \mathrm{En}$ face de la capitale, a la Pointe-de-Lévy, trois agglomérations se développent: l'une à l'est de la seigneurie de Lauzon; une deuxième entre ce qu'on appelle la "cabane des pères" et le fief Vilmé; la troisième sur la presqu'île que baignent l'Etchemin et la Chaudière. Entre les années 1670 et 1673, dans la seule seigneurie de Lauzon, l'on recense 45 concessions nouvelles. ${ }^{39} \mathrm{Au}$ souvenir du Québec aperçu en 1639, la Mère de l'Incarnation, émerveillée de ces progrès, écrit trente ans plus tard: "Lorsque

33. Pierre-Georges Roy, La Ville de Québec sous le régime français (2 vol., Québec, 1930), I: 331 . 236-303.

34. RAPQ (1930-1931), 100. Mémoire inédit de l'abbé Fénelon, AC, C IIA, 3:

35. RAPQ (1930-1931), 129.

36. RAPQ (1930-1931), 161.

37. Pierre-Georges Roy, Archives de la Province de Québec, Lettres de noblesse, généalogies... op. cit., I: 81.

38. Recensements du Canada, 1870-1871, IV: 11, 13. - Pierre-Georges Roy, L'Ile d'Orléans (Publié par la Commission des Monuments historiques de la Province de Québec) (Québec, 1928), 335-36.

39. J.-Ed. Roy, Histoire de la seigneurie de Lauzon (5 vol., Québec, 1897-1904), I: $180-181,291-294$. 
nous y sommes venues, il n'y avait que cinq ou six petites maisons tout au plus; tout le pays était de grandes forêts pleines de halliers. Maintenant Québec est une ville, au delà et aux environs de laquelle se trouvent quantité de bourgs et villages, dans une étendue de plus de cent lieues." 40 L'abbé Fénelon n'a pas moins admiré le paysage québécois. A la vue du superbe bassin qu'il apercevait de l'enclos du séminaire, il a écrit: "Tout le pays qui borde ce bassin et ces canaux, aussi bien que l'île d'Orléans est habité, en telle sorte que, de dessus cette montagne, où est Québec, on a le plaisir de voir des terres très belles et garnies de maisons, avec le bassin et les canaux, ce qui rend cette vue la plus belle du monde." 41

Le progrès paraît moins sensible dans la région trifluvienne. Des sauvages descendent encore le Saint-Maurice avec des chargements de pelleterie. Le poste perd toutefois de son importance commerciale, supplanté peu à peu par Montréal, depuis la paix iroquoise. Il n'en revient pas pour autant à la colonisation. Trois-Rivières n'aura pas été pour rien, pendant presque un demi-siècle, une sorte de capitale du castor. Trop d'habitants, premiers seigneurs et premiers colons, n'arrivent pas à se guérir de la course des bois. Le rôle des Trois-Rivières paraît avoir été de fournir des colons aux seigneuries des environs, au nord et au sud du Saint-Laurent, où de ses fils et d'anciens commandants du poste viennent de prendre des fiefs. C'est alors que les petits groupes de Champlain, de Batiscan, reçoivent des renforts et que commence à se développer Sainte-Anne-de-la-Pérade. ${ }^{4}{ }^{2}$ D'autres Trifluviens se risquent même vers le nord du lac Saint-Pierre où le fils aîné de Boucher possède la seigneurie de Grosbois ou Machiche. ${ }^{43}$ D'autres encore traversent le fleuve et donnent le premier essor à Nicolet. Mouest de Moras qui a été, en 1668, enseigne de Laubia aux TroisRivières, jette, dès 1669 , les bases d'un établissement sur les bords de la rivière Nicolet; et il fait appel aux colons des Trois-Rivières. A l'automne de 1670 le capitaine Arnauld de Laubia s'établit, aux

40. Lettres de la Révérende Mère Marie de l'Incarnation, éd. Richaudeau, II: 420.

41. Mémoire inédit de l'abbé Fénelon, 1671, AC, C IIA, 3: 236-303.

42. Raymond Douville, Les premiers seigneurs et colons de Sainte-Anne-de-laPérade, 1667-1681 (Les Trois-Rivières, 1946).

43. L'abbé Albert Tessier, 1535-1935-Trois-Rivières, Quatre siècles d'histoire. $2^{e}$ éd. (Les Trois-Rivières, 1935), 115. 
mêmes lieux, avec toute sa compagnie récemment amenée de France, soit une cinquantaine de colons. ${ }^{4}$ Tous ces soldats ne tarderont pas à déserter leurs concessions. Malgré tout, en 1672, Laubia récolte, sur son domaine à lui, trois à quatre cents minots de grain. ${ }^{45}$

A Montréal le défrichement marche à presque aussi grande allure que dans la région de Québec. Aussitôt conclue la paix iroquoise de 1666, et même aussitât connue l'arrivée prochaine des premières troupes de France, Ville-Marie, jusque là bourg aussi clos qu'une chartreuse, prend l'aspect d'une volière aux portes ouvertes. Les colons s'échappent de tous les côtés: vers le coteau Saint-Louis, vers la montagne, vers le pied du Courant, au-dessous de cet endroit, au dela de la rivière Saint-Pierre, tout le long de la rive du fleuve. L'arrivée des troupes accélère cet élan. Un véritable encerclement de l'île est en voie de s'accomplir. Les colons prennent d'abord pied, cela va de soi, le long du Saint-Laurent, grande voie de circulation, les uns au-dessus de la ville, les autres au-dessous. Diverses côtes s'alignent, en gagnant l'est de la ville: la côte Saint-François (la future Longue-Pointe), la côte Sainte-Anne, la côte Saint-Jean (future Pointe-aux-Trembles). Au-dessus de la ville, le long du fleuve toujours, d'autres colons prennent place entre la rivière Saint-Pierre et le Sault Saint-Louis. En face même du saut, le Rouennais René-Robert Cavelier de la Salle s'apprête, depuis 1667, à mettre en valeur un fief noble, terre élevée à cette dignité par M. de Queylus en 1669. La Salle n'est toutefois qu'un assez médiocre colonisateur. En 1669, lors de son départ pour la découverte de l'Ohio, il n'a encore défriché sur la partie principale de son fief, que huit ou neuf arpents; dans une autre partie de la seigneurie, le bois est simplement abattu et les bâtiments sont à peine commencés. ${ }^{4} 6$ Ce fief vient se joindre à d'autres de même qualité érigés depuis quelque temps. L'Hôtel-Dieu détient, depuis 1650 , un fief noble qui reçoit en 1666, puis en 1668, de notables agrandissements. ${ }^{47}$ Le major Closse a reçu de Maisonneuve pareille gratification qui se complétera d'une

44. Abbé Jos.-Elz. Bellemare, Histoire de Nicolet (Arthabaska, 1924), 32-37, $44-45$.

45. Mémoire de Talon à Colbert, 9 mars 1673, AC, F 4, 97.

46. Faillon, Histoire de la colonie française en Canada (3 vol., Villemarie, 1865), III: note, p. $288,289$.

47. Faillon, ibid., III: note, p. 225. 
autre en faveur de sa veuve. Un $M$. de Hautmesnil possède aussi son fief, depuis 1665 , entre le fleuve et la rivière des Prairies. ${ }^{4}$

Dans la création de ces premiers fiefs, l'on a toujours obéi au souci de défense militaire. Les mêmes motifs déterminent les Messieurs de Saint-Sulpice à entreprendre une nouvelle et grande distribution de fiefs. Faite pour une part, en même temps que celle de Talon, faut-il y voir l'inspiration de l'intendant? L'alerte iroquoise de 1668 à 1670 a pu suffire. Nulle part la menace ne devait secouer les esprits plus qu'à Montréal, cible toute désignée aux premiers coups des barbares. Organiser la défense des colons déjà trop éparpillés s'imposait avec urgence. Les Messieurs de Saint-Sulpice s'y mirent dès 1671. Et voilà qui explique l'apparition, sur le pourtour de l'île, de nombre de petits fiefs nobles. Pierre Picoté de Bélestre alla s'établir parmi les colons de la Pointe-aux-Trembles. ${ }^{49}$ Philippe de Carion Dufresnoy, lieutenant de la Compagnie de La Motte, au régiment de l'Estrade, Paul de Morel de Sainte-Hélène, enseigne de la même compagnie, prirent charge de l'entrée de la rivière de l'Assomption et d'une portion de la rive sur la rivière des Prairies ${ }^{50}$. Sur le fleuve et vers le centre de l'île, Zacharie du Puy, mis en possession de l'arrière-fief de Verdun, fit la liaison entre Ville-Marie et Cavelier de la Salle. ${ }^{51}$ Un autre point restait à pourvoir: le haut de l'Ile. Rive baignée par l'Outaouais, avec vue sur le lac des Deux-Montagnes, route fréquentée par les bandes iroquoises, la région tend à devenir de plus en plus le rendezvous des trafiquants et des convois de castor qui descendent de l'ouest. Quatre fiefs nobles y sont distribués en 1672; l'un à Michel-Sidrac du Gué, sieur de Boisbriant, le futur fief de Senneville $;^{52}$ un deuxième à Charles-Joseph d'Ailleboust des Musseaux $;^{53}$ un troisième, l'arrièrefief de Bellevue, conjointement aux frères Bertet: Louis de Bertet

48. Faillon, ibid., III: 337.

49. Faillon, ibid., III: 338.

50. P.-G. Roy, Archives de la Province de Québec, Inventaire des concessions en fiefs et seigneuries, fois et hommages et aveux et dénombrements conservés aux Archives de la Province de Québec (6 vol., Beauceville, 1927-1929), I: 190, 194.

51. Ibid., I: 193-194.

52. Ibid., I: 196.

53. Faillon, Histoire de la colonie française en Canada, op. cit., III: 341. 
de Chailly et Gabriel de Bertet de la Joubardière; ${ }^{54}$ un quatrième, à Claude Robutel de Saint-André, près de celui des fıères Bertet, jusqu'alors propriétaire du fief de la Noue en l'Ile Saint-Paul. ${ }^{55}$ Le caractère de ces concessions révèle bien l'intention des Messieurs de Saint-Sulpice. Aucun de ces fiefs ne se peut comparer pour l'étendue aux seigneuries concédées par Talon. Aucun ne dépasse 400 arpents, avec façade minime sur la rivière ou le fleuve. Aucun, non plus, n'est soumis à la sous-inféodation. Les Seigneurs de l'île n'ont donc pas eu pour principal dessein de placer ici et là des distributeurs de terres, mais bien plutôt d'organiser, sur tous les points stratégiques de l'île, des postes de défense au service des colons qui déjà ont pris terre en quelques endroits. ${ }^{56}$

A défaut du péril de guerre, le développement de la région eât imposé ces mesures. Le gouvernement de l'île a doublé sa population depuis 1667. De 760 âmes il est passé à 14 ou 1,500 âmes en $1672^{57}$. La ville elle-même sort résolument de son premier enclos. Elle escalade la côte qui avoisine la rive du fleuve, enjambe un gradin, tend vers une position plus stratégique. En même temps, elle s'allonge par les deux bouts. Pour entraîner les habitants à se construire sur la hauteur, les seigneurs y tracent en 1672 les premières rues, entre autres la rue Notre-Dame et la rue Saint-Jacques. ${ }^{58}$ Cette vie déborde l'île montréalaise. En 1670 Talon fait commencer sur l'Ile-Jésus, des travaux de défrichement aux frais de François Berthelot, secrétaire et commissaire général de l'artillerie, poudres et salpêtre de France. ${ }^{59}$ Les travaux marchent rondement. En 1674, le Père Dalmas y apercevait, sur la rive en face de l'île de Montréal, "diverses habitations." 60 Talon, qui rattache cet établissement à son plan de défense militaire contre l'Iroquois, y distribue des terres. Deux ans plus tard, la récolte du blé sur l'Ile-Jésus s'élève à 600 minots. Berthelot, qui y avait son manoir,

54. P.-G. Roy, Archives de la Province de Quebec, Inventaire... I: 195-96.

55. Ibid., I: 99-100. - Faillon, op. cit., III: 342.

56. Faillon, op. cit., III: 225, 228, 337, 338-42, 377.

57. Dollier de Casson, Histoire du Montréal, text and translation by Ralph Flenley (New York, 1928), 346.

58. Faillon, op. cit., III: 374-382, avec un plan de Ville-Marie.

59. P.-G. Roy, Archives de Québec, Lettres de noblesse, généalogies... I: 81.

60. RJ, LVIII: 116. 
se disait disposé à dépenser sur son domaine, encore 20,000 livres; il songeait en outre à y introduire une fabrique des poudres et salpêtres. Et il sollicitait, pour son fief, le titre de baronnie. ${ }^{61}$

Nul coin de la Nouvelle-France n'offre pourtant autant de nouveau que la péninsule du Richelieu. Toute cette région d'un sol si riche est enfin née à la vie. Ils sont là cinq capitaines des troupes qui "avec leurs subalternes et leurs compagnies", travaillent à former des "bourgades nouvelles". L'intendant, qui annonce la chose à Colbert, demande, en particulier pour Messieurs de Chambly et de Saurel, "quelque grâce du roi parce qu'ils travaillent fortement, à faire reussir les choses que je desire d'eux pour le service de Sa Majesté." 62 Les officiers de Carignan n'ont pas attendu leur blason de seigneurs pour se mettre au défrichement. Dès 1667 Talon a déjà distribué des terres a bon nombre d'entre eux "soubs le bon plaisir du Roy". Ces futurs seigneurs ont d'ailleurs sur d'autres le grand avantage de n'avoir pas à chercher longtemps ni loin leurs engagés ou censitaires: les soldats de leur compagnie les leur fournissent. Chance qui fut celle de tous les seigneurs militaires. En 1671 Colbert se disait heureux d'apprendre que tous les soldats passés au Canada l'année précédente, s'étaient "habitués" dans la seigneurie de leur capitaine. ${ }^{6}$ Dès cette même année 1667, au dire des Relations des Jésuites, l'on apercevait déjà, autour des forts du Richelieu, "des campagnes défrichées et couvertes de très-beau bled." 64 Jusque dans Québec, l'on s'entretient des merveilles qui s'accomplissent en ce coin de pays. Déja, nous apprend la Mère de l'Incarnation, des chemins relient les postes les uns aux autres; les officiers y ont de fort belles habitations, de vraies fermes avec bœufs, vaches, volailles, surtout MM. de Sorel et de Chambly. ${ }^{65}$ En 1672 Frontenac entend dire, de l'établissement de M. de Chambly, qu'elle est "la plus jolie de tout le pays". Verchères "travaille avec soin à son habitation." 66 Cette année-là deux compagnies tiennent encore gar-

61. RAPQ (1930-1931), 154, 173.

62. RAPQ (1930-1931), 132.

63. Lettre à Courcelle, 11 mars 1671, AC, B 3 (1671), 84.

64. RJ, L: 244.

65. RAPQ (1930-1931), 77-78. - Lettres de la Réverende Mère Marie de l'Incarnation... éd. Richaudeau, II: 351-352.

66. RAPQ (1926-1927), 17. 
nison au fort Saint-Louis. ${ }^{67}$ Dans les seigneuries de Saint-Ours, ${ }^{68}$ de Boucherville, de Longueuil, quelques échancrures de forêt ici et là, au bord du fleuve, attestent, là aussi, les premières prises du sol. ${ }^{69}$ A la Prairie de la Magdelaine, seigneurie des Jésuites, où un premier village s'ébauche en 1667 , plus de 40 concessions sont déjà faites au printemps de l'année suivante. ${ }^{70}$

Dans la Relation de 1668, un chroniqueur écrit. "Il fait beau voir a présent tous les rivages de nostre Fleuve de Saint-Laurent habités de nouvelles colonies qui vont s'estendant sur plus de quatre-vingt lieues de paîs le long des bords de cette grande Rivière, où l'on voit naître d'espace en espace de nouvelles Bourgades qui facilitent la navigation, la rendant plus agréable par la veuë de quantité de maisons, et plus commode par de fréquens lieux de repos." 71

Que n'eût pas écrit le même chroniqueur quatre ans plus tard? Le paysage de la Nouvelle-France s'est transformé. Le pays n'est plus comme en 1663 une colonie qui avait chance de vivre. Il est devenu une colonie vivante. Une race humaine l'étreint sur plus de 80 lieues. Que cet effort se fât poursuivi encore dix ans; que Talon eût réussi à maintenir sur leurs seigneuries et sur leurs lots, nouveaux seigneurs et nouveaux censitaires, et qu'il eat transformé en vrais défricheurs et terriens ces militaires au tempérament d'aventuriers, et le "grand royaume", la "grande monarchie" dont rêvait l'intendant, fut encore resté un mot, mais un mot plein d'avenir.

Lionel Groulx, ptre.

67. Mémoire inédit de l'abbé de Fénelon, 1671, AC, C IIA, 3: 236-303.

68. Du 5 au 7 novembre 1673 , M. de Saint-Ours accorde 26 concessions en sa seigneurie. Abbé Couillard-Després, Histoire de la seigneurie de Saint-Ours (2 vol., Montréal, 1917), I: 61-64.

69. Jodoin et Vincent, Histoire de Longueuil et de la famille de Longueuil (Montréal, 1889), 49-56.

70. Le Journal des Jésuites, publié par MM. les abbés Laverdière et Casgrain (Montréal, 1892), 361. - Rochemonteix, Les Jésuites et la Nouvelle-France au XVIIe siècle (5 vol., Paris, 1895-1896), III: 644.

71. RJ, LI: 166. 